\title{
Normalized solutions to Schrödinger systems with linear and nonlinear couplings*
}

\author{
Zhaoyang Yun ${ }^{a, b}$ Zhitao Zhang ${ }^{a, b, \dagger}$ \\ ${ }^{a}$ HLM, Academy of Mathematics and Systems Science, the Chinese \\ Academy of Sciences, Beijing 100190; \\ ${ }^{b}$ School of Mathematical Sciences, University of Chinese \\ Academy of Sciences, Beijing 100049, P.R. China
}

\begin{abstract}
In this paper, we study important Schrödinger systems with linear and nonlinear couplings

$$
\left\{\begin{array}{l}
-\Delta u_{1}-\lambda_{1} u_{1}=\mu_{1}\left|u_{1}\right|^{p_{1}-2} u_{1}+r_{1} \beta\left|u_{1}\right|^{r_{1}-2} u_{1}\left|u_{2}\right|^{r_{2}}+\kappa(x) u_{2} \text { in } \mathbb{R}^{N}, \\
-\Delta u_{2}-\lambda_{2} u_{2}=\mu_{2}\left|u_{2}\right|^{p_{2}-2} u_{2}+r_{2} \beta\left|u_{1}\right|^{r_{1}}\left|u_{2}\right|^{r_{2}-2} u_{2}+\kappa(x) u_{1} \text { in } \mathbb{R}^{N}, \\
u_{1} \in H^{1}\left(\mathbb{R}^{N}\right), u_{2} \in H^{1}\left(\mathbb{R}^{N}\right),
\end{array}\right.
$$

with the condition

$$
\int_{\mathbb{R}^{N}} u_{1}^{2}=a_{1}^{2}, \int_{\mathbb{R}^{N}} u_{2}^{2}=a_{2}^{2}
$$

where $N \geq 2, \mu_{1}, \mu_{2}, a_{1}, a_{2}>0, \beta \in \mathbb{R}, 2<p_{1}, p_{2}<2^{*}, 2<r_{1}+r_{2}<$ $2^{*}, \kappa(x) \in L^{\infty}\left(\mathbb{R}^{N}\right)$ with fixed sign and $\lambda_{1}, \lambda_{2}$ are Lagrangian multipliers. We use Ekland variational principle to prove this system has a normalized radially symmetric solution for $L^{2}$-subcritical case when $N \geq 2$, and use minimax method to prove this system has a normalized radially symmetric positive solution for $L^{2}$-supercritical case when $N=3, p_{1}=p_{2}=4, r_{1}=$ $r_{2}=2$.
\end{abstract}

Keywords: Nonlinear Schrödinger systems; Normalized solutions; Ekland variational principle; Minimax principle.

AMS Subject Classification(2010): 35J15, 35J47, 35J57

${ }^{*}$ Supported by National Natural Science Foundation of China(11771428, 12031015, 12026217), Email:yuanzhaoyang17@mails.ucas.ac.cn(Zhaoyang Yun); zzt@math.ac.cn(Zhitao Zhang)

${ }^{\dagger}$ Corresponding author 


\section{Introduction}

Schrödinger systems of the form which are related with Bose-Einstein condensates

$$
\left\{\begin{array}{l}
-\Delta u_{1}-\lambda_{1} u_{1}=f_{1}\left(u_{1}\right)+\partial_{1} F\left(u_{1}, u_{2}\right) \text { in } \mathbb{R}^{N} \\
-\Delta u_{2}-\lambda_{2} u_{2}=f_{2}\left(u_{2}\right)+\partial_{2} F\left(u_{1}, u_{2}\right) \text { in } \mathbb{R}^{N} \\
u_{1} \in H^{1}\left(\mathbb{R}^{N}\right), u_{2} \in H^{1}\left(\mathbb{R}^{N}\right)
\end{array}\right.
$$

where $N \geq 2$, have been concerned by many mathematicians in recent years. One motivation driving the search for (1.1) is to find the solutions of the timedependence system of coupled nonlinear Schrödinger equations.

In [2] T. Bartsch and L. Jeanjean studied the case when $f_{1}\left(u_{1}\right)=\mu_{1} u_{1}^{3}, f_{2}\left(u_{2}\right)=$ $\mu_{2} u_{2}^{3}, F\left(u_{1}, u_{2}\right)=\frac{1}{2} \beta u_{1}^{2} u_{2}^{2}, N=3$ together with the conditions

$$
\int_{\mathbb{R}^{3}} u_{1}^{2}=a_{1}^{2}, \int_{\mathbb{R}^{3}} u_{2}^{2}=a_{2}^{2},
$$

where $a_{1}, a_{2}, \mu_{1}, \mu_{2}, \beta>0, \lambda_{1}$ and $\lambda_{2}$ are Lagrange multipliers, they proved that there exists a $\beta_{1}>0$ depending on $a_{i}$ and $\mu_{i}$ such that if $0<\beta<\beta_{1}$ then (1.1)-(1.2) has a solution $\left(\lambda_{1}, \lambda_{2}, \bar{u}_{1}, \bar{u}_{2}\right)$ where $\lambda_{1}, \lambda_{2}<0$ and $\bar{u}_{1}$ and $\bar{u}_{2}$ are both postive and radially symmetric and there exists $\beta_{2}>0$ depending on $a_{i}$ and $\mu_{i}$ such that, if $\beta>\beta_{2}$, then (1.1)-(1.2) has a solution $\left(\lambda_{1}, \lambda_{2}, \bar{u}_{1}, \bar{u}_{2}\right)$ where $\lambda_{1}, \lambda_{2}<0$ and $\bar{u}_{1}$ and $\bar{u}_{2}$ are both positive and radially symmetric, other interesting results for normalized solutions can be found in [1], [3], [4], [5], [6], [10], [14], [16], [17], [18], [19], [23] and references therein.

In [13] K. Li and Z. T. Zhang studied the case when $\lambda_{1}$ and $\lambda_{2}$ are fixed and $f_{1}\left(u_{1}\right)=\mu_{1} u_{1}^{3}, f_{2}\left(u_{2}\right)=\mu_{2} u_{2}^{3}, F\left(u_{1}, u_{2}\right)=\frac{1}{2} \beta u_{1}^{2} u_{2}^{2}+\kappa u_{1} u_{2}$, where $\lambda_{1}, \lambda_{2}, \mu_{1}$, $\mu_{2}>0$ and $\beta, \kappa \in \mathbb{R}$, they proved that when $0<|\kappa|<\sqrt{\lambda_{1} \lambda_{2}}$ (1.1) has a solution $\left(u_{1}, u_{2}\right)$ such that $u_{1}, u_{2}>0$ if $\kappa>0$, and $u_{1}>0, u_{2}<0$ or $u_{1}<0, u_{2}>0$ if $\kappa<0$, other results of Schrödinger systems with linear and nonlinear couplings can be found in [15], [20], [21] etc. These papers inspire us to consider the Schrödinger systems with linear and nonlinear couplings

$$
\left\{\begin{array}{l}
-\Delta u_{1}-\lambda_{1} u_{1}=\mu_{1}\left|u_{1}\right|^{p_{1}-2} u_{1}+r_{1} \beta\left|u_{1}\right|^{r_{1}-2} u_{1}\left|u_{2}\right|^{r_{2}}+\kappa(x) u_{2} \text { in } \mathbb{R}^{N}, \\
-\Delta u_{2}-\lambda_{2} u_{2}=\mu_{2}\left|u_{2}\right|^{p_{2}-2} u_{2}+r_{2} \beta\left|u_{1}\right|^{r_{1}}\left|u_{2}\right|^{r_{2}-2} u_{2}+\kappa(x) u_{1} \text { in } \mathbb{R}^{N}, \\
u_{1} \in H^{1}\left(\mathbb{R}^{N}\right), u_{2} \in H^{1}\left(\mathbb{R}^{N}\right),
\end{array}\right.
$$


with the condition

$$
\int_{\mathbb{R}^{N}} u_{1}^{2}=a_{1}^{2}, \int_{\mathbb{R}^{N}} u_{2}^{2}=a_{2}^{2}
$$

Let $H^{1}\left(\mathbb{R}^{N}\right)$ be the usual Sobolev space and denote its norm by

$$
\|u\|:=\|u\|_{H_{r}^{1}}:=\left(|\nabla u|_{2}^{2}+|u|_{2}^{2}\right)^{1 / 2}
$$

In order to use the compact embedding in whole space, we denote the radially symmetric subspace as follows

$$
H_{r}^{1}:=H_{r a d}^{1}\left(\mathbb{R}^{N}\right):=\left\{u \in H^{1}\left(\mathbb{R}^{N}\right): u(x)=u(|x|)\right\} .
$$

We set

$$
S_{i}:=S_{a_{i}}:=\left\{u \in H_{r}^{1}:|u|_{2}=a_{i}\right\}, i=1,2,
$$

where $|u|_{p}:=|u|_{p, \mathbb{R}^{N}}:=\left(\int_{\mathbb{R}^{N}}|u|^{p}\right)^{1 / p}, p>1$. From standard variational arguments we know that critical points of the following functional on $S_{1} \times S_{2}$ are weak solutions of (1.3)- (1.4),

$$
\begin{aligned}
J\left(u_{1}, u_{2}\right)= & \frac{1}{2}\left(\int_{\mathbb{R}^{N}}\left|\nabla u_{1}\right|^{2}+\int_{\mathbb{R}^{N}}\left|\nabla u_{2}\right|^{2}\right)-\frac{\mu_{1}}{p_{1}}\left|u_{1}\right|_{p_{1}}^{p_{1}}-\frac{\mu_{2}}{p_{2}}\left|u_{2}\right|_{p_{2}}^{p_{2}} \\
& -\beta \int_{\mathbb{R}^{N}}\left|u_{1}\right|^{r_{1}}\left|u_{2}\right|^{r_{2}}-\int_{\mathbb{R}^{N}} \kappa(x) u_{1} u_{2} .
\end{aligned}
$$

We assume $\kappa(x)=\kappa(|x|), \kappa(x) \in L^{p}\left(\mathbb{R}^{N}\right)$ where $N / 2<p<\infty$ from the Palais's principle of symmetric criticality, the critical point of $\left.J\right|_{S_{1} \times S_{2}}$ on $H_{r}^{1} \times H_{r}^{1}$ is the critical point of $\left.J\right|_{S_{1} \times S_{2}}$ on $H^{1}\left(\mathbb{R}^{N}\right) \times H^{1}\left(\mathbb{R}^{N}\right)$. We just need to look for critical points of $\left.J\right|_{S_{1} \times S_{2}}$ on $H_{r}^{1} \times H_{r}^{1}$. By regularity theory of elliptic equations, weak solutions of (1.3)-(1.4) are classical. It is easy to see that $S_{1} \times S_{2}$ is a $C^{2}$ Finsler manifold modeled on the Hilbert space $H_{r}^{1} \times H_{r}^{1}$. We consider the functional $J\left(u_{1}, u_{2}\right)$ on the manifold $S_{1} \times S_{2}$, by Gagliardo-Nirenberg inequality we have that $J_{S_{1} \times S_{2}}$ is bounded from below for $L^{2}$-subcritical case: $2<p_{1}<2+4 / N, 2<p_{2}<$ $2+4 / N$ and $2<r_{1}+r_{2}<2+4 / N$, we will use minimizing method and Ekland variational principle to get a minimum point of $J_{S_{1} \times S_{2}}$. For $L^{2}$-supercritical case: $2+4 / N<p_{1}<2^{\star}, 2+4 / N<p_{2}<2^{\star}$ and $2+4 / N<r_{1}+r_{2}<2^{\star}$, where $2^{*}=2 N /(N-2)$, the functional $J_{S_{1} \times S_{2}}$ is not bounded from below, we try to construct a mountain pass structure of $J$ on the manifold $S_{1} \times S_{2}$ and by the minimax theory on the Finsler manifold which was introduced in [9] and to obtain the critical point of $J$ on $S_{1} \times S_{2}$. 
In section 2, we consider the $L^{2}$-subcritical case by Ekland variational principle on the manifold $S_{1} \times S_{2}$, by Liouville types theorems in [11], we obtain the following three theorems.

Theorem 1.1. Assume $\beta>0, \kappa(x)>0, \kappa(x)=\kappa(|x|)$ and $\kappa(x) \in L^{p}\left(\mathbb{R}^{N}\right) \cap$ $L^{\infty}\left(\mathbb{R}^{N}\right), N / 2<p<\infty$. If one of the following assumptions is satisfied:

(a) $2 \leq N \leq 4$ and $2<p_{1}, p_{2}, r_{1}+r_{2}<2+4 / N$, or

(b) $N \geq 5$ and $2<p_{1}, p_{2}<2+2 /(N-2)$ and $r_{1}+r_{2}<2+4 / N$.

Then (1.3)-(1.4) has a solution $\left(\lambda_{1,0}, \lambda_{2,0}, u_{1,0}, u_{2,0}\right)$ such that $\lambda_{1,0}, \lambda_{2,0}<0$ and $u_{1,0}, u_{2,0}>0$. Moreover, $u_{1,0}$ and $u_{2,0}$ are radially symmetric.

Similarly, when $\kappa(x)<0$ we obtain

Theorem 1.2. Assume $\beta>0, \kappa(x)<0, \kappa(x)=\kappa(|x|)$ and $\kappa(x) \in L^{p}\left(\mathbb{R}^{N}\right) \cap$ $L^{\infty}\left(\mathbb{R}^{N}\right), N / 2<p<\infty$. If one of the following assumptions is satisfied:

(a) $2 \leq N \leq 4$ and $2<p_{1}, p_{2}, r_{1}+r_{2}<2+4 / N$, or

(b) $N \geq 5$ and $2<p_{1}, p_{2}<2+2 /(N-2)$ and $2<r_{1}+r_{2}<2+4 / N$.

Then (1.3)-(1.4) has a solution $\left(\lambda_{1,0}, \lambda_{2,0}, u_{1,0}, u_{2,0}\right)$ such that $\lambda_{1,0}, \lambda_{2,0}<0$ and $u_{1,0}>0, u_{2,0}<0$ or $u_{1,0}<0, u_{2,0}>0$. Moreover, $u_{1,0}$ and $u_{2,0}$ are radially symmetric.

In section 3, we consider (1.3)-(1.4) when $N=3, p_{1}=p_{2}=4$, and $r_{1}=r_{2}=2$ which is $L^{2}-$ supercritical case.

$$
\left\{\begin{array}{l}
-\Delta u_{1}-\lambda_{1} u_{1}=\mu_{1} u_{1}^{3}+\beta u_{1} u_{2}^{2}+\kappa(x) u_{2} \text { in } \mathbb{R}^{3}, \\
-\Delta u_{2}-\lambda_{2} u_{2}=\mu_{2} u_{2}^{3}+\beta u_{1}^{2} u_{2}+\kappa(x) u_{1} \text { in } \mathbb{R}^{3}, \\
u_{1} \in H^{1}\left(\mathbb{R}^{3}\right), u_{2} \in H^{1}\left(\mathbb{R}^{3}\right),
\end{array}\right.
$$

with the condition

$$
\int_{\mathbb{R}^{3}} u_{1}^{2}=a_{1}^{2}, \int_{\mathbb{R}^{3}} u_{2}^{2}=a_{2}^{2},
$$

where $a_{1}, a_{2}, \mu_{1}, \mu_{2}>0, \beta \in \mathbb{R}$ and $\kappa(x) \in L^{\infty}\left(\mathbb{R}^{3}\right)$, (1.5)-(1.6) is the classical Bose-Einstein-condensates model.

By constructing the mountain pass structure on manifold $S_{1} \times S_{2}$, we have

Theorem 1.3. Assume $\beta>0, \kappa(x)>0, \kappa(x)=\kappa(|x|), \kappa(x) \in L^{p}\left(\mathbb{R}^{3}\right) \cap L^{\infty}\left(\mathbb{R}^{3}\right)$ for some $\frac{3}{2}<p<\infty, \frac{2}{3} \nabla \kappa(x) \cdot x+\kappa(x) \geq 0, \nabla \kappa(x) \cdot x$ is bounded and

$$
|\kappa(x)|_{\infty}<\frac{5}{18 C_{a_{1}, a_{2}}^{2} a_{1} a_{2}},
$$


where $C_{a_{1}, a_{2}}=\left(\left(\mu_{1}+\beta\right) a_{1} S^{4}+\left(\mu_{2}+\beta\right) a_{2} S^{4}\right)$ and $S$ denotes the Sobolev embedding constant in $\mathbb{R}^{3}$. Then (1.5)-(1.6) has a solution $\left(\bar{\lambda}_{1}, \bar{\lambda}_{2}, \bar{u}_{1}, \bar{u}_{2}\right)$ such that $\bar{\lambda}_{1}<0$, $\bar{\lambda}_{2}<0, \bar{u}_{1}>0, \bar{u}_{2}>0$. Moreover, $\bar{u}_{1}$ and $\bar{u}_{2}$ are radially symmetric.

Remark 1.1. From Theorem 1.3 we have that when $a_{1}$ or $a_{2}$ is small, then the upper bound of $\kappa(x)$ can be large.

Remark 1.2. For example we can take $\kappa(x)=\frac{c}{1+|x|^{\frac{3}{2}}}$ where $c<\frac{5}{18 C_{a_{1}, a_{2}}^{2} a_{1} a_{2}}$, which satisfies all conditions in Theorem 1.3 .

At the end of section 3, we will relax some restrictions of $\kappa(x)$ to obtain the same conclusion as Theorem 1.3.

\section{$2 \quad L^{2}$-subcritical case}

In this section, we prove Theorem 1.1 and Theorem 1.2 .

We assume $\kappa(x)$ satisfies $\kappa(x) \in L^{\infty}\left(\mathbb{R}^{N}\right), \kappa(x)>0$ or $\kappa(x)<0,2<p_{1}<$ $2+4 / N, 2<p_{2}<2+4 / N, 2<r_{1}+r_{2}<2+4 / N, \beta>0$, we work on the space $H_{r}^{1} \times H_{r}^{1}$, the corresponding energy functional of (1.3)-(1.4) on $S_{1} \times S_{2}$ is

$$
\begin{aligned}
J\left(u_{1}, u_{2}\right)= & \frac{1}{2}\left(\int_{\mathbb{R}^{N}}\left|\nabla u_{1}\right|^{2}+\int_{\mathbb{R}^{N}}\left|\nabla u_{2}\right|^{2}\right)-\frac{\mu_{1}}{p_{1}}\left|u_{1}\right|_{p_{1}}^{p_{1}}-\frac{\mu_{2}}{p_{2}}\left|u_{2}\right|_{p_{2}}^{p_{2}} \\
& -\beta \int_{\mathbb{R}^{N}}\left|u_{1}\right|^{r_{1}}\left|u_{2}\right|^{r_{2}}-\int_{\mathbb{R}^{N}} \kappa(x) u_{1} u_{2} .
\end{aligned}
$$

We try to find the critical point of $J$ on $S_{1} \times S_{2}$.

Lemma 2.1 (Gagliardo-Nirenberg inequality). For any $u \in H^{1}\left(\mathbb{R}^{N}\right)$ we have

$$
|u|_{p} \leq C_{N, p}|\nabla u|_{2}^{\alpha}|u|_{2}^{1-\alpha}
$$

where $\alpha=\frac{N(p-2)}{2 p}$.

Lemma 2.2. $J\left(u_{1}, u_{2}\right)$ is coercive and bounded from below on $S_{1} \times S_{2}$.

Proof. By lemma 2.1 we have

$$
\int_{\mathbb{R}^{N}}\left|u_{i}\right|^{p_{i}} \leq C\left(N, p_{i}, a_{i}\right)\left|\nabla u_{i}\right|_{2}^{\frac{N\left(p_{i}-2\right)}{2}}, i=1,2 .
$$


Set $C_{1}=C\left(N, p_{1}, a_{1}\right), C_{2}=C\left(N, p_{2}, a_{2}\right)$ and we can find $q, q^{\prime}$ such that $2 \leq r_{1} q$, $r_{2} q^{\prime} \leq 2^{*}, 1 / q+1 / q^{\prime}=1$, when $2 \leq r_{1}+r_{2} \leq 2^{*}$ we obtain

$$
\int_{\mathbb{R}^{N}}\left|u_{1}\right|^{r_{1}}\left|u_{2}\right|^{r_{2}} \leq\left|u_{1}\right|_{r_{1} q}^{r_{1}}\left|u_{2}\right|_{r_{2} q^{\prime}}^{r_{2}} \leq C\left|\nabla u_{1}\right|_{2}^{\frac{N\left(r_{1} q-2\right)}{2 q}}\left|\nabla u_{2}\right|_{2}^{\frac{N\left(r_{2} q^{\prime}-2\right)}{2 q^{\prime}}},
$$

where $C_{3}=C_{3}\left(N, a_{i}, r_{i}, q, q^{\prime}\right)$. By direct computation, when $r_{1}+r_{2}<2+4 / N$ we get that

$$
\frac{N\left(r_{1} q-2\right)}{2 q}+\frac{N\left(r_{2} q^{\prime}-2\right)}{2 q^{\prime}}<2 .
$$

By Young inequality we can find $\gamma_{1}, \gamma_{2}<2$ such that

$$
\int_{\mathbb{R}^{N}}\left|u_{1}\right|^{r_{1}}\left|u_{2}\right|^{r_{2}} \leq C_{3}\left(\left|\nabla u_{1}\right|^{\gamma_{1}}+\left|\nabla u_{2}\right|^{\gamma_{2}}\right) .
$$

By Hölder inequality, we get

$$
\int_{\mathbb{R}^{N}} \kappa(x) u_{1} u_{2} \leq|\kappa(x)|_{\infty} a_{1} a_{2}=: C_{4} .
$$

Thus we have for any $\left(u_{1}, u_{2}\right) \in S_{1} \times S_{2}$,

$$
\begin{aligned}
J\left(u_{1}, u_{2}\right) \geq & \frac{1}{2}\left(\left|\nabla u_{1}\right|_{2}^{2}+\left|\nabla u_{2}\right|_{2}^{2}\right)-\frac{C_{1} \mu_{1}}{p_{1}}\left|\nabla u_{1}\right|_{2}^{\frac{N\left(p_{1}-2\right)}{2}} \\
& -\frac{C_{2} \mu_{2}}{p_{2}}\left|\nabla u_{2}\right|_{2}^{\frac{N\left(p_{2}-2\right)}{2}}-C_{3} \beta\left(\left|\nabla u_{1}\right|_{2}^{\gamma_{1}}+\left|\nabla u_{2}\right|_{2}^{\gamma_{2}}\right)-C_{4},
\end{aligned}
$$

so $J\left(u_{1}, u_{2}\right)$ is coercive and bounded from below on $S_{1} \times S_{2}$.

We need some Liouville type theorems to ensure the weak limit of a PS sequence is not zero. If we assume $\kappa(x) \geq 0$ we can choose the minimizing sequence of $J$ on $S_{1} \times S_{2}$ is nonnegative.

Lemma 2.3 (See [11]). Assume that $u$ is a smooth function in $\mathbb{R}^{N}$,

(a) Suppose that $q \in(1, N /(N-2)]$ when $N \geq 3$ and $q \in(1, \infty)$ when $N=1,2$. Let $u \in L^{q}\left(\mathbb{R}^{N}\right)$ be a smooth nonnegative function satisfying $-\Delta u \geq 0$ in $\mathbb{R}^{N}$, then $u \equiv 0$.

(b) Suppose that $q \in(1,1+2 /(N-2)]$ the inequality $-\Delta u \geq u^{q}$ does not have a positive classical solution in $\mathbb{R}^{N}$. 
Lemma 2.4. Assume $\kappa(x) \geq 0$ and $\left(\lambda_{1}, \lambda_{2}, u_{1}, u_{2}\right) \in \mathbb{R}^{2} \times H_{r}^{1} \times H_{r}^{1}$ is a solution of (1.3), and assume $p_{1}, p_{2}<2+4 / N$ when $N \leq 4, p_{1}, p_{2}<2+N /(N-2)$ when $N \geq 5$, we have if $u_{1} \geq 0, u_{1} \not \equiv 0, u_{2} \geq 0$, then $\lambda_{1}<0$, if $u_{2} \geq 0, u_{2} \not \equiv 0$, $u_{1} \geq 0$, then $\lambda_{2}<0$.

Proof. In the first case when $u_{1} \not \equiv 0$, note that $\kappa(x) \geq 0$ and if $\lambda_{1} \geq 0$ we have

$$
-\Delta u_{1}=\lambda_{1} u_{1}+\mu_{1} u_{1}^{p_{1}-1}+r_{1} \beta u_{1}^{r_{1}-1} u_{2}^{r_{2}}+\kappa(x) u_{2} \geq 0
$$

then by Lemma 2.3 we can deduce that $u_{1} \equiv 0$ which is impossible, then $\lambda_{1}<0$. Similarly we can deduce the rest part of this lemma.

By Lemma 2.2 we have $J\left(u_{1}, u_{2}\right)$ is bounded from below and coercive on $S_{1} \times$ $S_{2}$ then we can find a minimizing sequence $\left\{\left(v_{1, n}, v_{2, n}\right)\right\}$ for $\left.J\right|_{S_{1} \times S_{2}}$ and because $\kappa(x) \geq 0$ we can assume $v_{i, n} \geq 0, i=1,2$. By Ekland variational principle we have $\left\{\left(u_{1, n}, u_{2, n}\right)\right\}$ is a PS sequence for $\left.J\right|_{S_{1} \times S_{2}}$ at level $c$, where

$$
c:=\inf _{S_{1} \times S_{2}} J\left(u_{1}, u_{2}\right)
$$

and $\left\|v_{i, n}-u_{i, n}\right\|_{H_{r}^{1}} \rightarrow 0$ as $n \rightarrow \infty$. Moreover, $\left\{\left(u_{1, n}, u_{2, n}\right)\right\}$ is bounded, then we have

$$
\left(u_{1, n}, u_{2, n}\right) \rightarrow\left(u_{1,0}, u_{2,0}\right) \in H_{r}^{1} \times H_{r}^{1},
$$

by standard arguments of compact embedding, we have $u_{i, 0} \geq 0, i=1,2$.

From above discussion we have

$$
\left.J\right|_{S_{1} \times S_{2}} ^{\prime}\left(u_{1, n}, u_{2, n}\right)=J^{\prime}\left(u_{1, n}, u_{2, n}\right)-\lambda_{1, n}\left(u_{1, n}, 0\right)-\lambda_{2, n}\left(0, u_{2, n}\right) \rightarrow 0
$$

in $\left(H_{r}^{1} \times H_{r}^{1}\right)^{*}$, where

$$
\begin{aligned}
\lambda_{1, n} & =\frac{1}{\left|u_{1, n}\right|_{2}^{2}}\left(J^{\prime}\left(u_{1, n}, u_{2, n}\right),\left(u_{1, n}, 0\right)\right) \\
& =\frac{1}{a_{1}^{2}}\left(\int_{\mathbb{R}^{N}}\left|\nabla u_{1, n}\right|^{2}-\mu_{1} \int_{\mathbb{R}^{N}}\left|u_{1, n}\right|^{p_{1}}-\beta \int_{\mathbb{R}^{N}}\left|u_{1, n}\right|^{r_{1}}\left|u_{2, n}\right|^{r_{2}}-\int_{\mathbb{R}^{N}} \kappa(x) u_{1, n} u_{2, n}\right), \\
\lambda_{2, n} & =\frac{1}{\left|u_{2, n}\right|_{2}^{2}}\left(J^{\prime}\left(u_{1, n}, u_{2, n}\right),\left(0, u_{2, n}\right)\right) \\
& =\frac{1}{a_{2}^{2}}\left(\int_{\mathbb{R}^{N}}\left|\nabla u_{2, n}\right|^{2}-\mu_{2} \int_{\mathbb{R}^{N}}\left|u_{2, n}\right|^{p_{2}}-\beta \int_{\mathbb{R}^{N}}\left|u_{1, n}\right|^{r_{1}}\left|u_{2, n}\right|^{r_{2}}-\int_{\mathbb{R}^{N}} \kappa(x) u_{1, n} u_{2, n}\right) .
\end{aligned}
$$


are bounded sequences and we may assume $\lambda_{1, n} \rightarrow \lambda_{1,0}, \lambda_{2, n} \rightarrow \lambda_{2,0}$ up to the subsequence. Then by weak convergence we have

$$
J^{\prime}\left(u_{1,0}, u_{2,0}\right)-\lambda_{1,0}\left(u_{1,0}, 0\right)-\lambda_{2,0}\left(0, u_{2,0}\right)=0 \text { in }\left(H_{r}^{1} \times H_{r}^{1}\right)^{*} .
$$

Moreover, $\left(\lambda_{1}, \lambda_{2}, u_{1,0}, u_{2,0}\right)$ is a solution of (1.3). In order to obtain $\left(u_{1,0}, u_{2,0}\right)$ also satisfies (1.4), we need the following lemma.

Lemma 2.5. If $\kappa(x) \geq 0, \kappa(x) \in L^{p}\left(\mathbb{R}^{N}\right)$ for some $N / 2<p<\infty$ and $\lambda_{i, 0}<0$ then $u_{i, n} \rightarrow u_{i, 0}, i=1,2$. As a consequence if $\lambda_{i, 0}<0, i=1,2$, then $\left(\lambda_{1,0}, \lambda_{2,0}, u_{1,0}, u_{2,0}\right)$ is a solution of (1.3) $-(1.4)$.

Proof. Because $\kappa(x) \in L^{p}\left(\mathbb{R}^{N}\right)$ where $p>N / 2$, then by the compact embedding and Hölder inequality we have

$$
\begin{gathered}
\int_{\mathbb{R}^{N}} \kappa(x) u_{1, n} u_{2, n} \rightarrow \int_{\mathbb{R}^{N}} \kappa(x) u_{1,0} u_{2,0}, \\
\left|u_{i, n}\right|_{p_{i}} \rightarrow\left|u_{i, 0}\right|_{p_{i}} i=1,2, \\
\int_{\mathbb{R}^{N}}\left|u_{1, n}\right|^{r_{1}}\left|u_{2, n}\right|^{r_{2}} \rightarrow \int_{\mathbb{R}^{N}}\left|u_{1,0}\right|^{r_{1}}\left|u_{2,0}\right|^{r_{2}},
\end{gathered}
$$

as $n \rightarrow \infty$. Notice that $\lambda_{1}<0$, by (2.2) and weak convergence we can deduce that

$$
\begin{gathered}
\left(J^{\prime}\left(u_{1, n}, u_{2, n}\right)-\lambda_{1,0}\left(u_{1, n}, 0\right),\left(u_{1, n}, 0\right)\right) \rightarrow 0 \\
\left(J^{\prime}\left(u_{1,0}, u_{2,0}\right)-\lambda_{1,0}\left(u_{1,0}, 0\right),\left(u_{1,0}, 0\right)\right)=0 .
\end{gathered}
$$

then from the above five equations we have that

$$
\left|\nabla u_{1, n}\right|_{2}^{2}-\lambda_{1,0}\left|u_{1, n}\right|_{2}^{2} \rightarrow\left|\nabla u_{1,0}\right|_{2}^{2}-\lambda_{1,0}\left|u_{1,0}\right|_{2}^{2}
$$

Because $\lambda_{1}<0$, we get $u_{1, n} \rightarrow u_{1,0}$ in $H_{r}^{1}$. Similarly if $\lambda_{2}<0$ we can get $u_{2, n} \rightarrow u_{2,0}$ in $H_{r}^{1}$. As a consequence when $\lambda_{1,0}$ and $\lambda_{2,0}$ are both negative, then $\left(\lambda_{1,0}, \lambda_{2,0}, u_{1,0}, u_{2,0}\right)$ is a solution of (1.3)-(1.4). Moreover, by maximum principle we have $u_{1,0}$ and $u_{2,0}$ are positive.

Next we consider the single equation which is useful in the following proof,

$$
-\Delta u+\lambda u=\mu|u|^{p-2} u \text { in } \mathbb{R}^{N},
$$


with the condition

$$
\int_{\mathbb{R}^{N}} u^{2}=a^{2}
$$

where $\mu>0,2<p<2+4 / N$ and $\lambda$ is Lagrangian multiplier. By Lemma 3.1 of [1], we know the corresponding energy functional of (2.3) on $S_{a}$ denotes by

$$
I(u):=I_{a, \mu}(u)=\frac{1}{2} \int_{\mathbb{R}^{N}}|\nabla u|^{2}-\frac{\mu}{p} \int_{\mathbb{R}^{N}}|u|^{p},
$$

and the least energy of $I$ on $S_{a}$ is denoted by

$$
m_{p}^{\mu}(a)=\inf _{S_{a}} I(u)
$$

which is achieved at some $u_{a} \in H_{r}^{1}$, and there exists $\lambda_{a}>0$ such that $\left(\lambda_{a}, u_{a}\right)$ is a solution of (2.3) and $\left|u_{a}\right|_{2}=a$. Moreover, $m_{p}^{\mu}(a)<0$.

Proof of Theorem 1.1. We denote the energy functional $\bar{J}$ for (1.3)-(1.4) on $S_{1} \times S_{2}$ when $\kappa(x)=0$, where

$$
\bar{J}\left(u_{1}, u_{2}\right)=\frac{1}{2}\left(\int_{\mathbb{R}^{N}}\left|\nabla u_{1}\right|^{2}+\int_{\mathbb{R}^{N}}\left|\nabla u_{2}\right|^{2}\right)-\frac{\mu_{1}}{p_{1}}\left|u_{1}\right|_{p_{1}}^{p_{1}}-\frac{\mu_{2}}{p_{2}}\left|u_{2}\right|_{p_{2}}^{p_{2}}-\beta \int_{\mathbb{R}^{N}}\left|u_{1}\right|^{r_{1}}\left|u_{2}\right|^{r_{2}} .
$$

Since $\beta>0$ and $\kappa(x) \geq 0$ we have $J\left(u_{1}, u_{2}\right) \leq \bar{J}\left(u_{1}, u_{2}\right)$, if $u_{1} \geq 0, u_{2} \geq 0$. Notice that $\bar{J}\left(u_{1}, u_{2}\right)=\bar{J}\left(\left|u_{1}\right|,\left|u_{2}\right|\right)$, we get

$$
\begin{aligned}
c:=\inf _{S_{1} \times S_{2}} J\left(u_{1}, u_{2}\right) & \leq \inf _{\substack{S_{1} \times S_{2} \\
u_{1}, u_{2} \geq 0}} J\left(u_{1}, u_{2}\right) \\
& \leq \inf _{\substack{S_{1} \times S_{2} \\
u_{1}, u_{2} \geq 0}} \bar{J}\left(u_{1}, u_{2}\right) \\
& =\inf _{S_{1} \times S_{2}} \bar{J}\left(u_{1}, u_{2}\right) \\
& \leq m_{p_{1}}^{\mu_{1}}\left(a_{1}\right)+m_{p_{2}}^{\mu_{2}}\left(a_{2}\right) \\
& <0 .
\end{aligned}
$$

Then for the weak limit $\left(u_{1,0}, u_{2,0}\right)$ of the PS sequence $\left\{\left(u_{1, n}, u_{2, n}\right)\right\}$ which is obtained in (2.1), we have the following four cases.

(i) If $\left(u_{1,0}, u_{2,0}\right)=(0,0)$, then by compact embedding we have

$$
0>c=\lim _{n \rightarrow \infty} J\left(u_{1, n}, u_{2, n}\right) \geq \liminf _{n \rightarrow \infty} \frac{1}{2}\left(\left|\nabla u_{1, n}\right|_{2}^{2}+\left|\nabla u_{2, n}\right|_{2}^{2}\right) \geq 0,
$$


which is impossible.

(ii) If $u_{1,0} \not \equiv 0$, but $u_{2,0} \equiv 0$, because $\left(\lambda_{1}, \lambda_{2}, u_{1,0}, u_{2,0}\right)$ is a solution of (1.3) then we must have $u_{1,0} \equiv 0$, because $\kappa(x)>0$, which is impossible.

(iii) If $u_{1,0} \equiv 0$ but $u_{2,0} \not \equiv 0$, it is same as (ii), which is impossible.

(iv) If $u_{1,0} \not \equiv 0$ and $u_{2,0} \not \equiv 0$, by Lemma 2.4 we have $\lambda_{1,0}, \lambda_{2,0}<0$, by Lemma 2.5, we have $u_{i, n} \rightarrow u_{i, 0}$ in $H_{r}^{1}$, by maximum principle we have $u_{1,0}, u_{2,0}>0$, then we finish the proof.

Now we consider the case $\kappa(x) \leq 0$.

Because $J$ is bounded from below and coercive, we can find a minimizing sequence $\left(v_{1, n}, v_{2, n}\right) \in S_{1} \times S_{2}$. Notice that $\kappa(x) \leq 0$ then without loss of generality we may assume $v_{1, n} \leq 0$ and $v_{2, n} \geq 0$, then by Ekland variational principle we have that there exists a PS sequence for $\left.J\right|_{S_{1} \times S_{2}}$ at level $c$, where

$$
c:=\inf _{S_{1} \times S_{2}} J\left(u_{1}, u_{2}\right)
$$

and $\left\|v_{i, n}-u_{i, n}\right\|_{H_{r}^{1}} \rightarrow 0, i=1,2$. By the coerciveness of $J$ on $S_{1} \times S_{2}$, there exists $\left(u_{1,0}, u_{2,0}\right) \in H_{r}^{1} \times H_{r}^{1}$ such that

$$
\left(u_{1, n}, u_{2, n}\right) \rightarrow\left(u_{1,0}, u_{2,0}\right) \text { in } H_{r}^{1} \times H_{r}^{1},
$$

in additional $u_{1,0} \leq 0, u_{2,0} \geq 0$. Similarly to prove Theorem 1.1 we can get $\lambda_{1,0}, \lambda_{2,0} \in \mathbb{R}$ such that $\left(\lambda_{1,0}, \lambda_{2,0}, u_{1,0}, u_{2,0}\right)$ is a solution of (1.3). We also have following lemma, which is similar to Lemma 2.4.

Lemma 2.6. Assume $\kappa(x) \leq 0$ and $\left(\lambda_{1}, \lambda_{2}, u_{1}, u_{2}\right) \in \mathbb{R}^{2} \times H_{r}^{1} \times H_{r}^{1}$ is a solution of (1.3), and assume $p_{1}, p_{2}<2+4 / N$ when $N \leq 4, p_{1}, p_{2}<2+N /(N-2)$ when $N \geq 5$, we have if $u_{1} \leq 0, u_{1} \not \equiv 0, u_{2} \geq 0$, then $\lambda_{1}<0$; if $u_{2} \geq 0, u_{2} \neq \equiv 0, u_{1} \leq 0$, then $\lambda_{2}<0$.

Proof. When $N \leq 4$, for the first case, we prove it by contradiction. If $\lambda_{1} \geq 0$, we have $-u_{1} \geq 0,-u_{1} \not \equiv 0$

$$
-\Delta\left(-u_{1}\right)=\lambda_{1}\left(-u_{1}\right)+\mu_{1}\left|u_{1}\right|^{p_{1}-2}\left(-u_{1}\right)+\beta r_{1}\left|u_{1}\right|^{r_{1}-2}\left(-u_{1}\right) u_{2}^{r_{2}}+\left(-\kappa(x) u_{2}\right) .
$$

So we get $-\Delta\left(-u_{1}\right) \geq 0$, by Lemma 2.3 we know $u_{1} \equiv 0$, which is impossible, thus $\lambda_{1}<0$. Similarly if $u_{2} \geq 0, u_{2} \not \equiv 0$ and $u_{1} \leq 0$, then $\lambda_{2}<0$. 
When $N \geq 5$ note that $-\Delta\left(-u_{1}\right) \geq \mu_{1}\left(-u_{1}\right)^{p_{1}-1}, u_{1} \leq 0$, and by Lemma 2.3 we have $u_{1} \equiv 0$, which is impossible. The rest part of the proof is the same as Lemma 2.4.

In order to obtain that $\left(u_{1,0}, u_{2,0}\right)$ in (2.4) satisfies (1.4), we need the following lemma.

Lemma 2.7. If $\kappa(x) \leq 0, \kappa(x) \in L^{p}\left(\mathbb{R}^{N}\right)$ for some $N / 2<p<\infty$ and $\lambda_{i, 0}<$ 0 , then $u_{i, n} \rightarrow u_{i, 0}, i=1,2$. As a consequence if $\lambda_{i, 0}<0, i=1,2$, then $\left(\lambda_{1,0}, \lambda_{2,0}, u_{1,0}, u_{2,0}\right)$ is a solution of (1.3)-(1.4).

Proof. Same as Lemma 2.5.

Now we prove the existence of solution for (1.3) $-(1.4)$ when $\kappa(x)<0$.

Proof of theorem 1.2. Using method of the proof of Theorem 1.1 and noticing that $\kappa(x) \leq 0$, we have

$$
\begin{aligned}
c:=\inf _{S_{1} \times S_{2}} J\left(u_{1}, u_{2}\right) & \leq \inf _{\substack{S_{1} \times S_{2} \\
u_{1} \leq 0, u_{2} \geq 0}} J\left(u_{1}, u_{2}\right) \\
& \leq \inf _{\substack{S_{1} \times S_{2} \\
u_{1} \leq 0, u_{2} \geq 0}} \bar{J}\left(u_{1}, u_{2}\right) \\
& =\inf _{S_{1} \times S_{2}} \bar{J}\left(u_{1}, u_{2}\right) \\
& \leq m_{p_{1}}^{\mu_{1}}\left(a_{1}\right)+m_{p_{2}}^{\mu_{2}}\left(a_{2}\right) \\
& <0 .
\end{aligned}
$$

Then we have following four cases.

(i) If $\left(u_{1,0}, u_{2,0}\right)=(0,0)$, then by compact embedding we have

$$
0>c=\lim _{n \rightarrow \infty} J\left(u_{1, n}, u_{2, n}\right) \geq \liminf _{n \rightarrow \infty} \frac{1}{2}\left(\left|\nabla u_{1, n}\right|_{2}^{2}+\left|\nabla u_{2, n}\right|_{2}^{2}\right) \geq 0
$$

which is impossible.

(ii) If $u_{1,0} \not \equiv 0$ but $u_{2,0} \equiv 0$, because $\left(\lambda_{1}, \lambda_{2}, u_{1,0}, u_{2,0}\right)$ is a solution of (1.3), then we must have $u_{1,0} \equiv 0$ because $\kappa(x)<0$, which is impossible.

(iii) If $u_{1,0} \equiv 0$ but $u_{2,0} \not \equiv 0$, the proof is same as (ii), it is impossible.

(iv) If $u_{1,0} \not \equiv 0$ and $u_{2,0} \not \equiv 0$, by Lemma 2.6, we have $\lambda_{1,0}, \lambda_{2,0}<0$. Then by Lemma $2.7 u_{i, n} \rightarrow u_{i, 0}$ in $H_{r}^{1}$, by maximum principle we have $u_{1,0}<0, u_{2,0}>$ 
0 . Finally if we take $\left\{\left(v_{1, n}, v_{2, n}\right)\right\}$ as the minimizing sequence of $\left.J\right|_{S_{1} \times S_{2}}$, such that $v_{1, n} \geq 0, v_{2, n} \leq 0$, then we can get $u_{1,0}>0, u_{2,0}<0$, and there exists $\left(\lambda_{1,0}, \lambda_{2,0}\right)$ such that $\left(\lambda_{1,0}, \lambda_{2,0}, u_{1,0}, u_{2,0}\right)$ is a solution of (1.3)-(1.4), then we finish the proof.

Remark 2.1. Moreover, by the properties of Schwarz rearrangement and $\beta>0$, we can easily deduce that the solution which is found in Theorem 1.1 and Theorem 1.2 is a ground state solution of (1.3) $-(\underline{1.4})$ in $H^{1}\left(\mathbb{R}^{N}\right) \times H^{1}\left(\mathbb{R}^{N}\right)$.

\section{$3 \quad L^{2}$-supercritical case}

In this section we consider the solutions for system (1.5)-(1.6) when $N=3, p_{1}=$ $p_{2}=4$ and $r_{1}=r_{2}=2$, which is $L^{2}$-supercritical case, the corresponding energy functional on $S_{1} \times S_{2}$ is defined by

$$
J\left(u_{1}, u_{2}\right)=\frac{1}{2} \int_{\mathbb{R}^{3}}\left|\nabla u_{1}\right|^{2}+\left|\nabla u_{2}\right|^{2}-\frac{1}{4} \int_{\mathbb{R}^{3}} \mu_{1} u_{1}^{4}+\mu_{2} u_{2}^{4}+2 \beta u_{1}^{2} u_{2}^{2}-\int_{\mathbb{R}^{3}} \kappa(x) u_{1} u_{2},
$$

where $\mu_{1}, \mu_{2}, \beta>0, \kappa(x)>0$ and $\kappa(x) \in L^{\infty}\left(\mathbb{R}^{3}\right) .\left.J\right|_{S_{1} \times S_{2}}$ is unbounded from below, so we can not achieve $\inf _{S_{1} \times S_{2}} J\left(u_{1}, u_{2}\right)$. In order to get the crtical point of $\left.J\right|_{S_{1} \times S_{2}}$, we will try to find a minimax value of $\left.J\right|_{S_{1} \times S_{2}}$, by constructing a mountain pass structure on $S_{1} \times S_{2}$. For this purpose we introduce the following two sets

$$
\begin{aligned}
& A_{K_{1}}:=\left\{\left(u_{1}, u_{2}\right) \in S_{1} \times S_{2}: \int_{\mathbb{R}^{3}}\left|\nabla u_{1}\right|^{2}+\left|\nabla u_{2}\right|^{2} \leq K_{1}\right\}, \\
& B_{K_{2}}:=\left\{\left(u_{1}, u_{2}\right) \in S_{1} \times S_{2}: \int_{\mathbb{R}^{3}}\left|\nabla u_{1}\right|^{2}+\left|\nabla u_{2}\right|^{2}=K_{2}\right\} .
\end{aligned}
$$

By Lemma 2.1 we have

$$
\int_{\mathbb{R}^{3}} \mu_{1} u_{1}^{4}+\mu_{2} u_{2}^{4}+2 \beta u_{1}^{2} u_{2}^{2} \leq C_{a_{1}, a_{2}}\left(\int_{\mathbb{R}^{3}}\left|\nabla u_{1}\right|^{2}+\left|\nabla u_{2}\right|^{2}\right)^{\frac{3}{2}},
$$

where $C_{a_{1}, a_{2}}=\left(\left(\mu_{1}+\beta\right) a_{1} S^{4}+\left(\mu_{2}+\beta\right) a_{2} S^{4}\right)$ and $S>0$ denotes the Sobolev embedding constant in $\mathbb{R}^{3}$.

Lemma 3.1. There exists $C_{1}>0$, where $C_{1}:=C_{1}\left(|\kappa(x)|_{\infty}, a_{1}, a_{2}\right)$ and $K_{1}>0$ such that for any $\left(u_{1}, u_{2}\right) \in A_{K_{1}}$

$$
J\left(u_{1}, u_{2}\right)>-C_{1} .
$$


Proof. We let $K_{1}<\frac{4}{C_{a_{1}, a_{2}}^{2}}$, where $\frac{4}{C_{a_{1}, a_{2}}^{2}}$ is the biggest zero point of the function

$$
\frac{1}{2} x-\frac{C_{a_{1}, a_{2}}}{4} x^{\frac{3}{2}}
$$

Then we have

$$
\begin{aligned}
J\left(u_{1}, u_{2}\right) & =\frac{1}{2} \int_{\mathbb{R}^{3}}\left|\nabla u_{1}\right|^{2}+\left|\nabla u_{2}\right|^{2}-\frac{1}{4} \int_{\mathbb{R}^{3}} \mu_{1} u_{1}^{4}+\mu_{2} u_{2}^{4}+\beta u_{1}^{2} u_{2}^{2}-\int_{\mathbb{R}^{3}} \kappa(x) u_{1} u_{2} \\
& \geq \frac{1}{2} \int_{\mathbb{R}^{3}}\left|\nabla u_{1}\right|^{2}+\left|\nabla u_{2}\right|^{2}-\frac{C_{a_{1}, a_{2}}}{4}\left(\int_{\mathbb{R}^{3}}\left|\nabla u_{1}\right|^{2}+\left|\nabla u_{2}\right|^{2}\right)^{\frac{3}{2}}-|\kappa(x)|_{\infty} a_{1} a_{2} \\
& \geq-|\kappa(x)|_{\infty} a_{1} a_{2},
\end{aligned}
$$

then we take $C_{1}=|\kappa(x)|_{\infty} a_{1} a_{2}$ to get (3.1).

Lemma 3.2. Assume $K_{2}=\frac{16}{9 C_{a_{1}, a_{2}}^{2}}$ and $|\kappa(x)|_{\infty}<\frac{5}{18 C_{a_{1}, a_{2}}^{2}}$, if $K_{1}$ small enough, then we have

$$
\sup _{A_{K_{1}}} J\left(u_{1}, u_{2}\right)<\inf _{B_{K_{2}}} J\left(u_{1}, u_{2}\right)
$$

and

$$
\inf _{B_{K_{2}}} J\left(u_{1}, u_{2}\right)>0 .
$$

Proof. Take $\left(v_{1}, v_{2}\right) \in B_{K_{2}},\left(u_{1}, u_{2}\right) \in A_{K_{1}}$, notice that $K_{2}>0$ is the maximum point of the function

$$
\frac{1}{2} x-\frac{C_{a_{1}, a_{2}}}{4} x^{\frac{3}{2}},
$$

$|\kappa(x)|_{\infty}<\frac{5}{18 C_{a_{1}, a_{2}}^{2}}$, and choose $K_{1}$ small enough, we have

$$
\begin{aligned}
J\left(v_{1}, v_{2}\right)-J\left(u_{1}, u_{2}\right)= & \frac{1}{2} \int_{\mathbb{R}^{3}}\left|\nabla v_{1}\right|^{2}+\left|\nabla v_{2}\right|^{2}-\frac{1}{2} \int_{\mathbb{R}^{3}}\left|\nabla u_{1}\right|^{2}+\left|\nabla u_{2}\right|^{2} \\
& -\frac{1}{4} \int_{\mathbb{R}^{3}} \mu_{1} v_{1}^{4}+\mu_{2} v_{2}^{4}+2 \beta v_{1}^{2} v_{2}^{2}+\frac{1}{4} \int_{\mathbb{R}^{3}} \mu_{1} u_{1}^{4}+\mu_{2} u_{2}^{4}+2 \beta u_{1}^{2} u_{2}^{2} \\
& -\int_{\mathbb{R}^{3}} \kappa(x) v_{1} v_{2}+\int_{\mathbb{R}^{3}} \kappa(x) u_{1} u_{2} \\
\geq & \frac{1}{2} \int_{\mathbb{R}^{3}}\left|\nabla v_{1}\right|^{2}+\left|\nabla v_{2}\right|^{2}-\frac{1}{2} \int_{\mathbb{R}^{3}}\left|\nabla u_{1}\right|^{2}+\left|\nabla u_{2}\right|^{2} \\
& -\frac{1}{4} \int_{\mathbb{R}^{3}} \mu_{1} v_{1}^{4}+\mu_{2} v_{2}^{4}+2 \beta v_{1}^{2} v_{2}^{2}-2|\kappa(x)|_{\infty} a_{1} a_{2} \\
\geq & \frac{1}{2} K_{2}-\frac{C_{a_{1}, a_{2}}}{4}\left(K_{2}\right)^{\frac{3}{2}}-\frac{1}{2} K_{1}-2|\kappa(x)|_{\infty} a_{1} a_{2} \\
> & 0 .
\end{aligned}
$$


Take $\left(u_{1}, u_{2}\right) \in B_{K_{2}}$, similarly to (3.4), we have

$$
\begin{aligned}
J\left(u_{1}, u_{2}\right) & =\frac{1}{2} \int_{\mathbb{R}^{3}}\left|\nabla u_{1}\right|^{2}+\left|\nabla u_{2}\right|^{2}-\frac{1}{4} \int \mu_{1} u_{1}^{4}+\mu_{2} u_{2}^{4}+2 \beta u_{1}^{2} u_{2}^{2}-\int_{\mathbb{R}^{3}} \kappa(x) u_{1} u_{2} \\
& \geq \frac{1}{2} K_{2}-\frac{C_{a_{1}, a_{2}}}{4}\left(K_{2}\right)^{\frac{3}{2}}-|\kappa(x)|_{\infty} a_{1} a_{2} \\
& >0 .
\end{aligned}
$$

This finishes the proof.

We fix a point $\left(v_{1}, v_{2}\right) \in A_{K_{1}}$ both nonnegative, and we try to find a point $\left(w_{1}, w_{2}\right)$ such that $J\left(w_{1}, w_{2}\right)$ is negative enough, and $\int_{\mathbb{R}^{3}}\left|\nabla w_{1}\right|^{2}+\left|\nabla w_{2}\right|^{2}$ is large enough. Then any path from $\left(v_{1}, v_{2}\right)$ to $\left(w_{1}, w_{2}\right)$ must pass through $B_{K_{2}}$, so we get a mountain pass structure on manifold $S_{1} \times S_{2}$. To do this, we use the translation which was firstly mentioned in [11],

$$
s \star u:=e^{\frac{3 s}{2}} u\left(e^{s} x\right),
$$

by direct calculation we have

$$
|s \star u|_{2}^{2}=|u|_{2}^{2}
$$

and

$$
|\nabla(s \star u)|_{2}^{2}=e^{2 s}|\nabla u|_{2}^{2}
$$

Moreover, we have

$$
\begin{aligned}
& J\left(s \star v_{1}, s \star v_{2}\right) \\
= & \frac{e^{2 s}}{2} \int_{\mathbb{R}^{3}}\left|\nabla v_{1}\right|^{2}+\left|\nabla v_{2}\right|^{2}-\frac{e^{3 s}}{4} \int_{\mathbb{R}^{3}} \mu_{1} v_{1}^{4}+\mu_{2} v_{2}^{4}+2 \beta v_{1}^{2} v_{2}^{2}-\int_{\mathbb{R}^{3}} \kappa(x)\left(s \star v_{1}\right)\left(s \star v_{2}\right) \\
\leq & \frac{e^{2 s}}{2} \int_{\mathbb{R}^{3}}\left|\nabla v_{1}\right|^{2}+\left|\nabla v_{2}\right|^{2}-\frac{e^{3 s}}{4} \int_{\mathbb{R}^{3}}\left(\mu_{1} v_{1}^{4}+\mu_{2} v_{2}^{4}+2 \beta v_{1}^{2} v_{2}^{2}\right)+|\kappa(x)|_{\infty} a_{1} a_{2} .
\end{aligned}
$$

If $s$ is large enough, then we have $J\left(s \star v_{1}, s \star v_{2}\right)<-C_{1}$, where $C_{1}$ is defined in (3.1), and we take $\left(w_{1}, w_{2}\right):=\left(s \star v_{1}, s \star v_{2}\right)$.

Then we can get a mountain pass structure of $J$ on manifold $S_{1} \times S_{2}$

$$
\Gamma:=\left\{\gamma(t)=\left(\gamma_{1}(t), \gamma_{2}(t)\right): \gamma(0)=\left(v_{1}, v_{2}\right), \gamma(1)=\left(w_{1}, w_{2}\right)\right\}
$$

and the mountain pass value is

$$
c:=\inf _{\gamma \in \Gamma} \sup _{t \in[0,1]} J(\gamma(t)) \geq \inf _{B_{K_{2}}} J\left(u_{1}, u_{2}\right)>0 .
$$


In order to obtain the boundedness of the PS sequence at mountain pass value $c$ we use the following notations

$$
\tilde{J}\left(s, u_{1}, u_{2}\right):=J\left(s \star u_{1}, s \star u_{2}\right)=\tilde{J}\left(0, s \star u_{1}, s \star u_{2}\right),
$$

the corresponding minimax structure of $\tilde{J}$ on $\mathbb{R} \times S_{1} \times S_{2}$ as follows

$$
\tilde{\Gamma}:=\left\{\tilde{\gamma}(t)=\left(s(t), \gamma_{1}(t), \gamma_{2}(t)\right): \tilde{\gamma}(0)=\left(0, v_{1}, v_{2}\right), \tilde{\gamma}(1)=\left(0, w_{1}, w_{2}\right)\right\},
$$

and it minimax value is

$$
\tilde{c}=\inf _{\tilde{\gamma} \in \tilde{\Gamma}} \sup _{t \in[0,1]} \tilde{J}(\tilde{\gamma}(t)) .
$$

First we claim that $\tilde{c}=c$.

In fact, from $\tilde{\Gamma} \supset \Gamma$ we have $\tilde{c} \leq c$. On the other hand, for any

$$
\tilde{\gamma}(t)=\left(s(t), \gamma_{1}(t), \gamma_{2}(t)\right)
$$

by definition we have

$$
\tilde{J}(\tilde{\gamma}(t))=J(s(t) \star \gamma(t)),
$$

and $s(t) \star \gamma(t) \in \Gamma$ is obvious, then

$$
\sup _{t \in[0,1]} \tilde{J}(\tilde{\gamma}(t)) \geq \inf _{\gamma \in \Gamma} \sup _{t \in[0,1]} J(\gamma(t))
$$

by definition of $\tilde{c}$ we have $\tilde{c} \geq c$, then $\tilde{c}=c$. Because

$$
\tilde{J}\left(s, u_{1}, u_{2}\right)=\tilde{J}\left(0, s \star u_{1}, s \star u_{2}\right),
$$

we take a sequence $\tilde{\gamma}_{n}=\left(0, \gamma_{1, n}, \gamma_{2, n}\right) \in \tilde{\Gamma}$ such that

$$
c=\lim _{n \rightarrow \infty} \sup _{t \in[0,1]} \tilde{J}\left(\tilde{\gamma}_{n}(t)\right) .
$$

Moreover, using the fact that $\kappa(x)>0$ we have

$$
\tilde{J}\left(s,\left|u_{1}\right|,\left|u_{2}\right|\right) \leq \tilde{J}\left(s, u_{1}, u_{2}\right),
$$

then we can assume $\gamma_{1, n}, \gamma_{2, n} \geq 0$. By Theorem 3.2 in [9] (it is easy to check the conditions of Theorem 3.2 in [9] are satisfied by Lemma 3.2) we can get a PS sequence $\left(s_{n}, \tilde{u}_{1, n}, \tilde{u}_{2, n}\right)$ of $\tilde{J}$ on $\mathbb{R} \times S_{1} \times S_{2}$ at level c. Moreover,

$$
\lim _{n \rightarrow \infty}\left|s_{n}\right|+\operatorname{dist}_{H_{r}^{1}}\left(\left(\tilde{u}_{1, n}, \tilde{u}_{2, n}\right),\left(\gamma_{1, n}, \gamma_{2, n}\right)\right)=0 .
$$


So we have $s_{n} \rightarrow 0$ and $\tilde{u}_{1, n}^{-}, \tilde{u}_{2, n}^{-} \rightarrow 0$ in $H_{r}^{1}$, where and in the following $u^{-}(x):=$ $\min \{u(x), 0\}$ and $u^{+}(x)=\max \{u(x), 0\}$, then by taking

$$
\left(u_{1, n}, u_{2, n}\right):=\left(s_{n} \star \tilde{u}_{1, n}, s_{n} \star \tilde{u}_{2, n}\right),
$$

we have the following lemma.

Lemma 3.3. $\left(u_{1, n}, u_{2, n}\right)$ is a PS sequence of $J\left(u_{1}, u_{2}\right)$ at level $c$ on $S_{1} \times S_{2}$.

Proof. First we know that $\left(s_{n}, \tilde{u}_{1, n}, \tilde{u}_{2, n}\right)$ is a PS sequence of $\tilde{J}\left(s, u_{1}, u_{2}\right)$, then for any $\left(\phi_{1}, \phi_{2}\right) \in H_{r}^{1} \times H_{r}^{1}$ we have

$$
\begin{aligned}
& \tilde{J}_{\mathbf{u}}^{\prime}\left(s_{n}, \tilde{u}_{1, n}, \tilde{u}_{2, n}\right)\left(\phi_{1}, \phi_{2}\right) \\
= & e^{2 s_{n}} \int_{\mathbb{R}^{3}} \nabla \tilde{u}_{1, n} \cdot \nabla \phi_{1}+\nabla \tilde{u}_{2, n} \cdot \nabla \phi_{2} \\
& -e^{3 s_{n}} \int_{\mathbb{R}^{3}} \mu_{1} \tilde{u}_{1, n}^{3} \phi_{1}+\mu_{2} \tilde{u}_{2, n}^{3}+\beta \tilde{u}_{1, n} \phi_{1} \tilde{u}_{2, n}^{2}+\beta \tilde{u}_{1, n}^{2} \tilde{u}_{2, n} \phi_{2} \\
& -\int_{\mathbb{R}^{3}} \kappa\left(e^{-s_{n}} x\right) \tilde{u}_{1, n} \phi_{2}-\int_{\mathbb{R}^{3}} \kappa\left(e^{-s_{n}} x\right) \tilde{u}_{2, n} \phi_{1} \\
= & \int_{\mathbb{R}^{3}} \nabla u_{1, n} \cdot \nabla\left(s_{n} \star \phi_{1}\right)+\nabla u_{2, n} \cdot \nabla\left(s_{n} \star \phi_{2}\right) \\
& -\int_{\mathbb{R}^{3}} \mu_{1} u_{1, n}^{3}\left(s_{n} \star \phi_{1}\right)+\mu_{2} u_{2, n}^{3}\left(s_{n} \star \phi_{1}\right)+\beta u_{1, n}^{2} u_{2, n}\left(s_{n} \star \phi_{2}\right)+\beta u_{1, n} u_{2, n}^{2}\left(s_{n} \star \phi_{1}\right) \\
& -\int_{\mathbb{R}^{3}} \kappa(x) u_{1, n}\left(s_{n} \star \phi_{2}\right)-\int_{\mathbb{R}^{3}} \kappa(x) u_{2, n}\left(s_{n} \star \phi_{1}\right) \\
= & J^{\prime}\left(u_{1, n}, u_{2, n}\right)\left(s_{n} \star \phi_{1}, s_{n} \star \phi_{2}\right),
\end{aligned}
$$

where $\mathbf{u}=\left(u_{1}, u_{2}\right)$. Notice that $-s \star(s \star \phi)=\phi, \forall s \in \mathbb{R}$, we have

$$
\tilde{J}_{\mathbf{u}}^{\prime}\left(s_{n}, \tilde{u}_{1, n}, \tilde{u}_{2, n}\right)\left(-s_{n} \star \phi_{1},-s_{n} \star \phi_{2}\right)=J^{\prime}\left(u_{1, n}, u_{2, n}\right)\left(\phi_{1}, \phi_{2}\right) .
$$

It is obvious that $\left(\phi_{1}, \phi_{2}\right) \in T_{\left(u_{1, n}, u_{2, n}\right)} S_{1} \times S_{2}$ if and only if $\left(-s_{n} \star \phi_{1},-s_{n} \star \phi_{2}\right) \in$ $T_{\left(\tilde{u}_{1, n}, \tilde{u}_{2, n}\right)} S_{1} \times S_{2}$ see [5]. Since $s_{n} \rightarrow 0$, we have $-s_{n} \star \phi_{i} \rightarrow \phi_{i}, i=1,2$ as $n \rightarrow \infty$ in $H_{r}^{1}$, then for $n$ large enough there exist $A_{1}>0$ and $A_{2}>0$ such that

$$
A_{1}<\frac{\left\|\left(\phi_{1}, \phi_{2}\right)\right\|}{\left\|\left(-s_{n} \star \phi_{1},-s_{n} \star \phi_{2}\right)\right\|}<A_{2},
$$

where $\left(\phi_{1}, \phi_{2}\right) \neq(0,0)$. Let $\|\cdot\|_{\star}$ be the norm of the cotangent space $\left(T_{\left(u_{1}, u_{2}\right)} S_{1} \times\right.$ $\left.S_{2}\right)^{\star}$. Thus for any $\left(\phi_{1}, \phi_{2}\right) \in T_{\left(u_{1, n}, u_{2, n}\right)} S_{1} \times S_{2}$ and $\left(\phi_{1}, \phi_{2}\right) \neq(0,0)$, we have

$$
|J|_{S_{1} \times S_{2}}^{\prime}\left(u_{1, n}, u_{2, n}\right) \frac{\left(\phi_{1}, \phi_{2}\right)}{\left\|\left(-s_{n} \star \phi_{1},-s_{n} \star \phi_{2}\right)\right\|} \mid \leq\left\|\left(\left.\tilde{J}\right|_{S_{1} \times S_{2}}\right)_{\mathbf{u}}^{\prime}\left(s_{n}, \tilde{u}_{1, n}, \tilde{u}_{2, n}\right)\right\|_{\star} \rightarrow 0,
$$


as $n \rightarrow \infty$. Take the supremum both side and notice (3.8), we have

$$
A_{1}\left\|\left.J\right|_{S_{1} \times S_{2}} ^{\prime}\left(u_{1, n}, u_{2, n}\right)\right\|_{\star} \leq\left\|\left(\left.\tilde{J}\right|_{S_{1} \times S_{2}}\right)_{\mathbf{u}}^{\prime}\left(s_{n}, \tilde{u}_{1, n}, \tilde{u}_{2, n}\right)\right\|_{\star} \rightarrow 0, \text { as } n \rightarrow \infty \text {. }
$$

From the fact that $A_{1}>0$ we have

$$
\left\|\left.J\right|_{S_{1} \times S_{2}} ^{\prime}\left(u_{1, n}, u_{2, n}\right)\right\|_{\star} \rightarrow 0, \text { as } n \rightarrow \infty
$$

On the other hand we have

$$
J\left(u_{1, n}, u_{2, n}\right)=\tilde{J}\left(s_{n}, \tilde{u}_{1, n}, \tilde{u}_{2, n}\right) \rightarrow c, \text { as } n \rightarrow \infty
$$

This finishes the proof.

Lemma 3.4. If $\kappa(x)$ and $\nabla \kappa(x) \cdot x$ is bounded in $\mathbb{R}^{3}$, then the PS sequence $\left(u_{1, n}, u_{2, n}\right)$ obtained in Lemma 3.3 of $J\left(u_{1}, u_{2}\right)$ on $S_{1} \times S_{2}$ at level $c$ is bounded in $H_{r}^{1} \times H_{r}^{1}$.

Proof. Since $\left(s_{n}, \tilde{u}_{1, n}, \tilde{u}_{2, n}\right)$ is a PS sequence for $\tilde{J}$, we have

$$
\frac{d}{d s} \tilde{J}\left(s_{n}, \tilde{u}_{1, n}, \tilde{u}_{2, n}\right) \rightarrow 0,
$$

i.e.,

$$
\begin{aligned}
& \int_{\mathbb{R}^{3}}\left|\nabla u_{1, n}\right|^{2}+\left|\nabla u_{2, n}\right|^{2}-\frac{3}{4} \int_{\mathbb{R}^{3}} \mu_{1} u_{1, n}^{4}+\mu_{2} u_{2, n}^{4}+2 \beta u_{1, n}^{2} u_{2, n}^{2} \\
& +\int_{\mathbb{R}^{3}} \nabla \kappa\left(e^{-s_{n}} x\right) \cdot e^{-s_{n}} x \tilde{u}_{1, n} \tilde{u}_{2, n} \rightarrow 0 .
\end{aligned}
$$

On the other hand, notice that $\tilde{J}\left(s_{n}, \tilde{u}_{1, n}, \tilde{u}_{2, n}\right)=J\left(u_{1, n}, u_{2, n}\right)$, we obtain

$$
\begin{aligned}
J\left(u_{1, n}, u_{2, n}\right)= & \frac{1}{2} \int_{\mathbb{R}^{3}}\left|\nabla u_{1, n}\right|^{2}+\left|\nabla u_{2, n}\right|^{2}-\frac{1}{4} \int_{\mathbb{R}^{3}} \mu_{1} u_{1, n}^{4}+\mu_{2} u_{2, n}^{4}+2 \beta u_{1, n}^{2} u_{2, n}^{2} \\
& -\int_{\mathbb{R}^{3}} \kappa\left(e^{-s_{n}} x\right) \tilde{u}_{1, n} \tilde{u}_{2, n} \rightarrow c,
\end{aligned}
$$

then use the boundness of $\kappa(x), \nabla \kappa(x) \cdot x,\left(\tilde{u}_{1, n}, \tilde{u}_{2, n}\right) \in S_{1} \times S_{2}$, (3.9) and (3.10), we can deduce that $\int_{\mathbb{R}^{3}}\left|\nabla u_{1, n}\right|^{2}+\left|\nabla u_{2, n}\right|^{2}$ is bounded, notice that $\left(u_{1, n}, u_{2, n}\right) \in S_{1} \times S_{2}$, we get $\left(u_{1, n}, u_{2, n}\right)$ is bounded in $H_{r}^{1} \times H_{r}^{1}$. 
Because $\left(u_{1, n}, u_{2, n}\right)$ is bounded in $H_{r}^{1} \times H_{r}^{1}$, then there exists $\left(\bar{u}_{1}, \bar{u}_{2}\right) \in H_{r}^{1} \times H_{r}^{1}$ such that

$$
\left(u_{1, n}, u_{2, n}\right) \rightarrow\left(\bar{u}_{1}, \bar{u}_{2}\right), \text { in } H_{r}^{1} \times H_{r}^{1} .
$$

Lemma 3.5. Under the assumptions of Lemma 3.4, and we assume $\frac{1}{3} \nabla \kappa(x) \cdot x+$ $\kappa(x) \geq 0$, then there exists $C>0$ such that for $n$ large we have

$$
\left|\nabla u_{1, n}\right|_{2}^{2}+\left|\nabla u_{2, n}\right|_{2}^{2} \geq C
$$

Proof. By (3.9) and $\tilde{u}_{1, n}^{-}, \tilde{u}_{2, n}^{-} \rightarrow 0$ in $H_{r}^{1}$, we have

$$
\begin{aligned}
& \int_{\mathbb{R}^{3}}\left|\nabla u_{1, n}\right|^{2}+\left|\nabla u_{2, n}\right|^{2}-\frac{3}{4} \int_{\mathbb{R}^{3}} \mu_{1} u_{1, n}^{4}+\mu_{2} u_{2, n}^{4}+2 \beta u_{1, n}^{2} u_{2, n}^{2} \\
& +\int_{\mathbb{R}^{3}} \nabla \kappa\left(e^{-s_{n}} x\right) \cdot e^{-s_{n}} x \tilde{u}_{1, n}^{+} \tilde{u}_{2, n}^{+}=o(1),
\end{aligned}
$$

and

$$
\begin{aligned}
& \frac{1}{2} \int_{\mathbb{R}^{3}}\left|\nabla u_{1, n}\right|^{2}+\left|\nabla u_{2, n}\right|-\frac{1}{4} \int_{\mathbb{R}^{3}} \mu_{1} u_{1, n}^{4}+\mu_{2} u_{2, n}^{4}+2 \beta u_{1, n}^{2} u_{2, n}^{2} \\
& \quad-\int_{\mathbb{R}^{3}} \kappa\left(e^{-s_{n}} x\right) \tilde{u}_{1, n}^{+} \tilde{u}_{2, n}^{+}=c+o(1) .
\end{aligned}
$$

By (3.6), we have $c>0$, thus

$$
\begin{aligned}
c+o(1) & =J\left(u_{1, n}, u_{2, n}\right) \\
& =\frac{1}{6} \int_{\mathbb{R}^{3}}\left|\nabla u_{1, n}\right|^{2}+\left|\nabla u_{2, n}\right|^{2}-\int_{\mathbb{R}^{3}}\left(\frac{1}{3} \nabla \kappa\left(e^{-s_{n}} x\right) \cdot e^{-s_{n}} x+\kappa\left(e^{-s_{n}} x\right)\right) \tilde{u}_{1, n}^{+} \tilde{u}_{2, n}^{+} \\
& \leq \frac{1}{6} \int_{\mathbb{R}^{3}}\left|\nabla u_{1, n}\right|^{2}+\left|\nabla u_{2, n}\right|^{2},
\end{aligned}
$$

then for $n$ large enough and take $C=3 c$, this finishes the proof.

Because $\left(u_{1, n}, u_{2, n}\right)$ is a PS sequence of $J$ on $S_{1} \times S_{2}$, for any $\left(\phi_{1}, \phi_{2}\right) \in H_{r}^{1} \times H_{r}^{1}$, there exists $\lambda_{1, n}, \lambda_{2, n}$ such that as $n \rightarrow \infty$

$$
\begin{aligned}
& \left(\left.J\right|_{S_{1} \times S_{2}} ^{\prime}\left(u_{1, n}, u_{2, n}\right),\left(\phi_{1}, \phi_{2}\right)\right) \\
= & \int_{\mathbb{R}^{3}} \nabla u_{1, n} \nabla \phi_{1}+\int_{\mathbb{R}^{3}} \nabla u_{2, n} \nabla \phi_{2}-\mu_{1} \int_{\mathbb{R}^{3}} u_{1, n}^{3} \phi_{1}-\mu_{2} \int_{\mathbb{R}^{3}} u_{2, n}^{3} \phi_{2} \\
& -\beta \int_{\mathbb{R}^{3}} u_{1, n} u_{2, n}^{2} \phi_{1}-\beta \int_{\mathbb{R}^{3}} u_{1, n}^{2} u_{2, n} \phi_{2}-\int_{\mathbb{R}^{3}} \kappa(x) u_{1, n} \phi_{2} \\
& -\int_{\mathbb{R}^{3}} \kappa(x) u_{2, n} \phi_{1}-\lambda_{1, n} \int_{\mathbb{R}^{3}} u_{1, n} \phi_{1}-\lambda_{2, n} \int_{\mathbb{R}^{3}} u_{2, n} \phi_{2} \\
= & o\left(\left\|\left(\phi_{1}, \phi_{2}\right)\right\|\right) .
\end{aligned}
$$


From the proof of Theorem 1.1, taking $p_{1}=p_{2}=4$ and $r_{1}=r_{2}=2$ we have

$$
\begin{aligned}
& \lambda_{1, n} a_{1}^{2}=\int_{\mathbb{R}^{3}}\left|\nabla u_{1, n}\right|^{2}-\mu_{1} \int_{\mathbb{R}^{3}} u_{1, n}^{4}-\beta \int_{\mathbb{R}^{3}} u_{1, n}^{2} u_{2, n}^{2}-\int_{\mathbb{R}^{3}} \kappa(x) u_{1, n} u_{2, n}, \\
& \lambda_{2, n} a_{2}^{2}=\int_{\mathbb{R}^{3}}\left|\nabla u_{2, n}\right|^{2}-\mu_{2} \int_{\mathbb{R}^{3}} u_{2, n}^{4}-\beta \int_{\mathbb{R}^{3}} u_{1, n}^{2} u_{2, n}^{2}-\int_{\mathbb{R}^{3}} \kappa(x) u_{1, n} u_{2, n},
\end{aligned}
$$

then it is easy to deduce that $\left\{\lambda_{1, n}\right\}$ and $\left\{\lambda_{2, n}\right\}$ are bounded. So we may assume

$$
\begin{aligned}
\lambda_{1, n} & \rightarrow \bar{\lambda}_{1}, \\
\lambda_{2, n} & \rightarrow \bar{\lambda}_{2},
\end{aligned}
$$

by choosing subsequence if necessary.

Lemma 3.6. Under the conditions of Lemma [3.5, assume $\frac{2}{3} \nabla \kappa(x) \cdot x+\kappa(x) \geq 0$ and $\kappa(x)>0$, then at least one of $\bar{\lambda}_{i}, i=1,2$ is negative.

Proof. Notice that $\tilde{u}_{1, n}^{-} \rightarrow 0, \tilde{u}_{2, n}^{-} \rightarrow 0$ in $H_{r}^{1}$, (3.12), (3.13) and (3.9), we have

$$
\begin{aligned}
& \bar{\lambda}_{1} a_{1}^{2}+\bar{\lambda}_{2} a_{2}^{2} \\
= & \lambda_{1, n} a_{1}^{2}+\lambda_{2, n} a_{2}^{2}+o(1) \\
= & \int_{\mathbb{R}^{3}}\left|\nabla u_{1, n}\right|^{2}+\left|\nabla u_{2, n}\right|^{2}-\int_{\mathbb{R}^{3}} \mu_{1} u_{1, n}^{4}+\mu_{2} u_{2, n}^{4}+2 \beta u_{1, n}^{2} u_{2, n}^{2}-2 \int_{\mathbb{R}^{3}} \kappa(x) u_{1, n} u_{2, n}+o(1) \\
= & -\frac{1}{3} \int_{\mathbb{R}^{3}}\left|\nabla u_{1, n}\right|^{2}+\left|\nabla u_{2, n}\right|^{2}-\left(\int_{\mathbb{R}^{3}}\left(\frac{4}{3} \nabla \kappa\left(e^{-s_{n}} x\right) \cdot e^{-s_{n}} x+2 \kappa\left(e^{-s_{n}} x\right)\right) \tilde{u}_{1, n}^{+} \tilde{u}_{2, n}^{+}\right)+o(1) \\
\leq & -\frac{1}{3} \int_{\mathbb{R}^{3}}\left|\nabla u_{1, n}\right|^{2}+\left|\nabla u_{2, n}\right|^{2}+o(1) \\
< & -\frac{1}{3} C+o(1),
\end{aligned}
$$

then one of the $\bar{\lambda}_{1}, \bar{\lambda}_{2}$ is negative.

Proof of Theorem 1.3. From standard argument we can conclude that $\left(\bar{\lambda}_{1}, \bar{\lambda}_{2}, \bar{u}_{1}, \bar{u}_{2}\right)$ is a solution of the system

$$
\left\{\begin{array}{l}
-\Delta u_{1}-\lambda_{1} u_{1}=\mu_{1} u_{1}^{3}+\beta u_{1} u_{2}^{2}+\kappa(x) u_{2} \text { in } \mathbb{R}^{3}, \\
-\Delta u_{2}-\lambda_{2} u_{2}=\mu_{2} u_{2}^{3}+\beta u_{1}^{2} u_{2}+\kappa(x) u_{1} \text { in } \mathbb{R}^{3}, \\
u_{1} \in H^{1}\left(\mathbb{R}^{3}\right), u_{2} \in H^{1}\left(\mathbb{R}^{3}\right),
\end{array}\right.
$$


where $\bar{u}_{1}$ and $\bar{u}_{2}$ are nonnegative, then we just need to prove $\left(u_{1, n}, u_{2, n}\right) \rightarrow\left(\bar{u}_{1}, \bar{u}_{2}\right)$ strongly in $H_{r}^{1} \times H_{r}^{1}$. By (3.11) together with compact embedding and $\lambda_{1, n} \rightarrow \bar{\lambda}_{1}$ we have

$$
\begin{aligned}
o(1) & =\left(J^{\prime}\left(u_{1, n}, u_{2, n}\right)-J^{\prime}\left(\bar{u}_{1}, \bar{u}_{2}\right),\left(u_{1, n}-\bar{u}_{1}, 0\right)\right)-\bar{\lambda}_{1} \int_{\mathbb{R}^{3}}\left(u_{1, n}-\bar{u}_{1}\right)^{2} \\
& =\int_{\mathbb{R}^{3}}\left|\nabla\left(u_{1, n}-\bar{u}_{1}\right)\right|^{2}-\bar{\lambda}_{1}\left(u_{1, n}-\bar{u}_{1}\right)^{2}+o(1) .
\end{aligned}
$$

As the proof of Lemma 2.5 we just need to show that $\bar{\lambda}_{1}, \bar{\lambda}_{2}<0$ to obtain the strong convergence. When $\kappa(x) \in L^{p}\left(\mathbb{R}^{3}\right)$ for some $\frac{3}{2}<p<\infty$. By Lemma 3.6 without loss of generality we may assume $\bar{\lambda}_{1}<0$ if $\bar{\lambda}_{2} \geq 0$, then we have

$$
-\Delta \bar{u}_{2}=\bar{\lambda}_{2} \bar{u}_{2}+\mu_{1} \bar{u}_{2}^{3}+\beta \bar{u}_{1}^{2} \bar{u}_{2}+\kappa(x) \bar{u}_{1} \geq 0,
$$

by Lemma 2.3 , we have $\bar{u}_{2} \equiv 0$. But $u_{1, n} \rightarrow \bar{u}_{1}$ in $H_{r}^{1}$, thus $\left|\bar{u}_{1}\right|_{2}^{2}=a_{1}^{2}$, so $\bar{u}_{1} \not \equiv 0$ and $\left(\bar{u}_{1}, 0\right)$ can not be the solution of (1.5)), therefore $\bar{\lambda}_{2}<0$. From maximum principle we can get $\bar{u}_{1}, \bar{u}_{2}>0$, then we finish the proof of Theorem 1.3 ,

Remark 3.1. Because $\kappa(x)>0$, from $\frac{2}{3} \nabla \kappa(x) \cdot x+\kappa(x) \geq 0$ we can deduce that $\frac{1}{3} \nabla \kappa(x) \cdot x+\kappa(x) \geq 0$, so we just need assume $\frac{2}{3} \nabla \kappa(x) \cdot x+\kappa(x) \geq 0$ in Theorem 1.3 .

Moreover, from the proof of Theorem 1.3 we can get a stronger theorem. First we introduce a new condition

$\left(K_{1}\right)$ There exist $T_{1}, T_{2}>0$ such that

$$
T_{1}+2 T_{2}<\frac{1}{108 C_{a_{1}, a_{2}}^{2} a_{1} a_{2}} \leq \frac{\inf _{B_{K_{2}}} J}{2 a_{1} a_{2}} \leq \frac{c}{2 a_{1} a_{2}},
$$

where $c$ is the mountain pass value defined in (3.6) and

$$
\nabla \kappa(x) \cdot x \geq \max \left\{-3 T_{1},-\frac{3}{2} T_{2}\right\}
$$

Theorem 3.1. Assume that $\kappa(x)>0, \kappa(x)=\kappa(|x|), \kappa(x) \in L^{p}\left(\mathbb{R}^{3}\right) \cap L^{\infty}\left(\mathbb{R}^{3}\right)$ where $\frac{3}{2}<p<\infty, \nabla \kappa(x) \cdot x$ is bounded. Moreover, if $\kappa(x)$ satisfies $\left(K_{1}\right)$ and $|\kappa(x)|_{\infty}<\frac{5}{18 C_{a_{1}, a_{2}}^{2}}$, then system (1.5) $-(1.6)$ has a solution $\left(\bar{\lambda}_{1}, \bar{\lambda}_{2}, \bar{u}_{1}, \bar{u}_{2}\right)$. Moreover, $\bar{u}_{1}>0, \bar{u}_{2}>0$ are radial and $\bar{\lambda}_{1}<0, \bar{\lambda}_{2}<0$. 
Proof. We just need to show that one of the limits of $\left\{\lambda_{1, n}\right\}$ and $\left\{\lambda_{2, n}\right\}$ is negative. By $\left(K_{1}\right)$ and $\kappa(x)>0$ we have

$$
\begin{aligned}
& \frac{1}{3} \nabla \kappa(x) \cdot x+\kappa(x) \geq-T_{1}, \\
& \frac{2}{3} \nabla \kappa(x) \cdot x+\kappa(x) \geq-T_{2},
\end{aligned}
$$

and from above two equations we have

$$
\begin{aligned}
c+o(1) & =J\left(u_{1, n}, u_{2, n}\right) \\
& \leq \frac{1}{6} \int_{\mathbb{R}^{3}}\left|\nabla u_{1, n}\right|^{2}+\left|\nabla u_{2, n}\right|^{2}-\int_{\mathbb{R}^{3}}\left(\frac{1}{3} \nabla \kappa\left(e^{-s_{n}} x\right) \cdot e^{-s_{n}} x+\kappa\left(e^{-s_{n}} x\right)\right) \tilde{u}_{1, n}^{+} \tilde{u}_{2, n}^{+} \\
& \leq \frac{1}{6} \int_{\mathbb{R}^{3}}\left|\nabla u_{1, n}\right|^{2}+\left|\nabla u_{2, n}\right|^{2}+T_{1} a_{1} a_{2},
\end{aligned}
$$

then we have for $n$ large $\frac{1}{6} \int_{\mathbb{R}^{3}}\left|\nabla u_{1, n}\right|^{2}+\left|\nabla u_{2, n}\right|^{2}>\frac{c}{3}$.

$$
\begin{aligned}
& \lambda_{1} a_{1}^{2}+\lambda_{2} a_{2}^{2} \\
= & \lambda_{1, n} a_{1}^{2}+\lambda_{2, n} a_{2}^{2}+o(1) \\
= & \int_{\mathbb{R}^{3}}\left|\nabla u_{1, n}\right|^{2}+\left|\nabla u_{2, n}\right|^{2}-\int_{\mathbb{R}^{3}} \mu_{1} u_{1, n}^{4}+\mu_{2} u_{2, n}^{4}+2 \beta u_{1, n}^{2} u_{2, n}^{2}-2 \int_{\mathbb{R}^{3}} \kappa(x) u_{1, n} u_{2, n}+o(1) \\
= & -\frac{1}{3} \int_{\mathbb{R}^{3}}\left|\nabla u_{1, n}\right|^{2}+\left|\nabla u_{2, n}\right|^{2}-\left(\int_{\mathbb{R}^{3}}\left(\frac{4}{3} \nabla \kappa\left(e^{-s_{n}} x\right) \cdot e^{-s_{n}} x+2 \kappa\left(e^{-s_{n}} x\right)\right) \tilde{u}_{1, n}^{+} \tilde{u}_{2, n}^{+}\right)+o(1) \\
\leq & -\frac{1}{3} \int_{\mathbb{R}^{3}}\left|\nabla u_{1, n}\right|^{2}+\left|\nabla u_{2, n}\right|^{2}+2 T_{2} a_{1} a_{2}+o(1) \\
< & -\frac{2}{3} c+\frac{1}{2} c+o(1) \\
= & -\frac{1}{6} c+o(1),
\end{aligned}
$$

we know that one of $\bar{\lambda}_{i}, i=1,2$ is negative, following the steps of the proof of Theorem 1.3, we can get Theorem 3.1 .

Remark 3.2. From above arguments we can relax the condition $\kappa(x)>0$ to $\kappa(x) \geq 0$ and $\kappa(x) \not \equiv 0$ or $\kappa(x)<0$ to $\kappa(x) \leq 0$ and $\kappa(x) \not \equiv 0$ in Theorem 1.1 , Theorem 1.2, Theorem 1.3 and Theorem 3.1. In fact, if $\bar{u}_{2} \equiv 0$, then $\kappa(x) \bar{u}_{1} \equiv 0$ and $\bar{u}_{1}$ solves the equation

$$
-\Delta u-\lambda_{1} u=\mu_{1} u^{3} \text { in } \mathbb{R}^{3},
$$

where $\lambda_{1}<0$ (see the proof of Theorem 1.3). It has a unique positive solution up to translations. So $\kappa(x) \bar{u}_{1} \equiv 0$ is impossible, thus $\bar{u}_{2} \not \equiv 0$. 


\section{References}

[1] T. Bartsch, L. Jeanjean, Normalized solutions for nonlinear Schrödinger systems, Proc. Roy. Soc. Edinburgh Sect. A 148 (2018), no. 2, 225-242.

[2] T. Bartsch, S. de Valeriola, Normalized solutions of nonlinear Schrödinger equations. Arch. Math. (Basel) 100 (2013), no. 1, 75-83.

[3] T. Bartsch, L. Jeanjean, N. Soave, Normalized solutions for a system of coupled cubic Schrödinger equations on $\mathbb{R}^{3}$. J. Math. Pures Appl. 106 (2016), no. 4, 583-614.

[4] T. Bartsch, N. Soave, A natural constraint approach to normalized solutions of nonlinear Schrödinger equations and systems. J. Funct. Anal. 272 (2017), no. $12,4998-5037$.

[5] T. Bartsch, N. Soave, Multiple normalized solutions for a competing system of Schrödinger equations. Calc. Var. Partial Differential Equations 58 (2019), no. 1, Art. 22, 24 pp.

[6] T. Bartsch, N. Soave, Correction to: "A natural constraint approach to normalized solutions of nonlinear Schrödinger equations and systems" [J. Funct. Anal. 272 (12) (2017) 4998-5037] [MR3639521]. J. Funct. Anal. 275 (2018), no. $2,516-521$.

[7] Benedetta Noris, Hugo Tavares and Gianmaria Verzini, Normalized solutions for nonlinear Schrödinger systems on bounded domains, Nonlinearity 32 (2019), 1044-1072

[8] Deconinck B., Kevrekidis P. G., Nistazakis H. E., Frantzeskakis D.J., Linearly coupled Bose-Einstein condensates: From Rabi oscillations and quasiperiodic solutions to oscillating domain walls and spiral waves, Physical Review A, 70(6) (2004), 063605.

[9] N. Ghoussoub. Duality and perturbation methods in critical point theory, Cambridge Tracts in Mathematics, vol. 107, Cambridge University Press, 1993.

[10] Tianxiang Gou, Zhitao Zhang, Normalized solutions to the Chern-SimonsSchrödinger system. J. Funct. Anal. 280 (2021), no. 5, 108894, 65pp. 
[11] N. Ikoma. Compactness of minimizing sequences in nonlinear Schrödinger systems under multiconstraint conditions. Adv. Nonlin. Studies 14 (2014), 115136.

[12] L. Jeanjean, Existence of solutions with prescribed norm for semilinear elliptic equations. Nonlinear Anal. 28 (1997), no. 10, 1633-1659.

[13] K. Li, Z. T. Zhang, Existence of solutions for a Schrödinger system with linear and nonlinear couplings, J. Math. Phys. 57 (8) (2016), 081504, 17 pp.

[14] Haijun Luo, Zhitao Zhang, Normalized solutions to the fractional Schrödinger equations with combined nonlinearities. Calc. Var. Partial Differential Equations 59 (2020), no. 4, Paper No. 143, 35 pp.

[15] H. J. Luo, Z. T. Zhang, Existence and nonexistence of bound state solutions for Schrödinger systems with linear and nonlinear couplings, J. Math. Anal. Appl. 475 (2019), 350-363

[16] B. Noris, H. Tavares, G. Verzini, Existence and orbital stability of the ground states with prescribed mass for the $L^{2}$-critical and supercritical NLS on bounded domains. Anal. PDE 7 (2014), no. 8, 1807-1838.

[17] B. Noris, H. Tavares, G. Verzini, Stable solitary waves with prescribed $L^{2}$ mass for the cubic Schrödinger system with trapping potentials. Discrete Contin. Dyn. Syst. 35 (2015), no. 12, 6085-6112.

[18] B. Noris, H. Tavares, G. Verzini, Normalized solutions for nonlinear Schrödinger systems on bounded domains. Nonlinearity 32 (2019), no. 3, 10441072.

[19] D. Pierotti, G. Verzini, Normalized bound states for the nonlinear Schrödinger equation in bounded domains. Calc. Var. Partial Differential Equations 56 (2017), no. 5, Art. 133, 27 pp.

[20] R.S. Tian, Z.T. Zhang, Existence and bifurcation of solutions for a double coupled system of Schrödinger equations, Sci. China Math. 58 (8) (2015), 1607 1620. 
[21] X. Zhang and Z. Zhang, Distribution of positive solutions to Schrödinger systems with linear and nonlinear couplings, J. Fixed Point Theory Appl., 22(2) (2020), 33,21pp.

[22] Z.T. Zhang, Variational, topological, and partial order methods with their applications, Springer, Heidelberg, 2013.

[23] X.P. Zhu, H.S. Zhou, Bifurcation from the essential spectrum of superlinear elliptic equations. Appl. Anal. 28 (1988), no. 1, 51-66. 